\title{
MODELING OF JOINT SIGNAL DETECTION AND PARAMETER ESTIMATION ON THE BACKGROUND OF NON-GAUSSIAN NOISE
}

Volodimir Palahin, Vitaliy Filipov, Serhii Leleko, Oleksandr Ivchenko, Olena Palahina

\author{
Cherkasy State Technological University \\ Cherkasy, Ukraine \\ Leleko@i.ua
}

\begin{abstract}
The paper presents the results and describes the process of modeling of a system of joint signal detection and parameter estimation on the background of a non-Gaussian noise based on moment and cumulant description of random variables, polynomials of Kunchenko and moment quality criterion type of Neyman-Pearson.
\end{abstract}

Keywords: non-Gaussian noise, moment and cumulant representation of random variables, truncated stochastic polynom, polynomial solving rules

\section{Introduction}

Detection of signals and estimation of their parameters is an important task to be solved in electronic, telecommunication, information and other systems $[1,2]$. Typically, these tasks are solved on the basis of the assumption that the signals are observed on the background of Gaussian noise [3]. The widespread use of the normal distribution of random variables in many cases does not reflect the real processes. This paper considers a different approach to the construction of models and methods of detection and estimation of signal parameters on the background of non-Gaussian noise, based on the application of moment-cumulant representation of random variables [4] and stochastic polynomials of Kunchenko [5].

\section{Solution of the problem}

The aim of this work is the simulation modeling of processes of joint detection of signals and estimation of their parameters on the background of non-Gaussian noise on the basis of moment-cumulant representation of random variables $\xi$, polynomial decision rules (DR) of detected signals and the use of truncated stochastic polynomials for their assessment. 
Suppose we have a sample volume of $n$ identically distributed random values $X=\left\{x_{1}, x_{2}, \ldots x_{n}\right\}$ from an entire assembly of values of random variables $\xi$. This random variable is an additive mixture of the informative signal and stationary non-Gaussian noise: $\xi(t)=S(t, \vartheta)+\eta(t)$. The useful signal $S(t, \vartheta)$ is represented as a single rectangular video pulse with a random time of receipt $\tau$, duration $T$, time lag $t$.

For the signal detection and the estimation of its parameter, we use nonlinear methods $[5,6]$, which are based on moment-cumulant representation of a random variable $\xi$. We consider a model of the process of detection of joint signal $S_{9}$ and estimation of parameter $\vartheta$ for the degrees of the polynomial $s=2,3$.

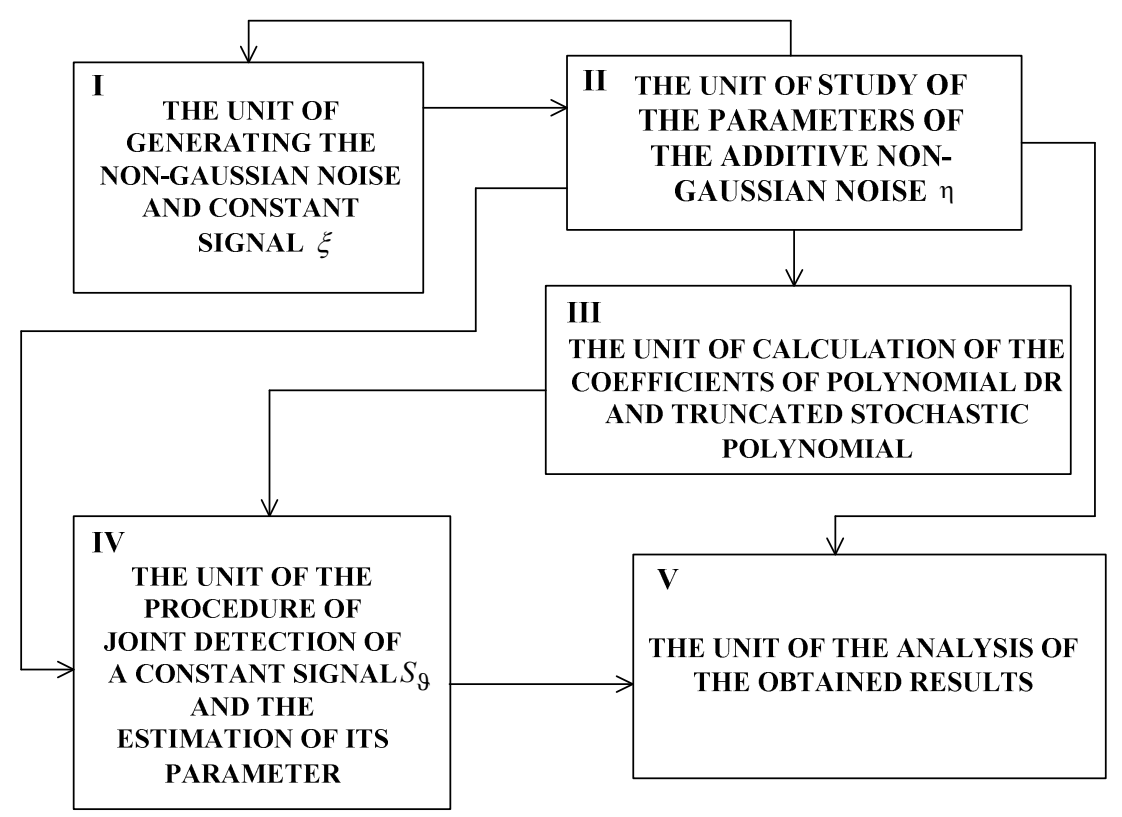

Fig. 1. Block diagram of the simulation modeling

The structural scheme of the simulation modeling is represented in Figure 1. The simulation modeling consists of five stages: the generation of non-Gaussian noise and the constant signal (unit I); study of the parameters of the additive nonGaussian noise (unit II); the calculation of the coefficients of polynomial DR and truncated stochastic polynomial (unit III); the procedure of joint detection of a constant signal and the estimation of its parameter (unit IV); the analysis of the obtained results (unit V).

The first phase involves the generation of nonsymmetrical non-Gaussian noise with a trivial expectation function and variance $\chi_{2}$, where the cumulant coefficients from the fourth to the sixth order equal zero $\left(\gamma_{4}=\gamma_{5}=\gamma_{6}=0\right)$, and the coef- 
ficient of skewness $\gamma_{3}$ varies within its definition field [5]. Cumulant coefficients of the seventh and higher orders take arbitrary values.

The second stage involves the estimation of parameters of nonsymmetrical interference $\eta$. The procedure of preliminary estimation of parameters of interference $\eta$ is necessary to check the values of noise variance $\chi_{2}$ and skewness $\gamma_{3}$, which were asked at the first stage of modeling in the generating unit $\xi$. Based on the obtained values of the parameter estimates of additive nonsymmetrical interference at the third stage of the simulation, there is the calculation of mathematical expectations of DR within the hypothesis and the alternative, correlates, the coefficients of the truncated stochastic polynomial and polynomial DR [6].

The centered correlates $\mathrm{F}\left(H_{0}\right)_{\mathrm{i}, \mathrm{j}\{k k\}}$ and $\mathrm{F}\left(H_{1}\right)_{\mathrm{i}, \mathrm{j}\}\{k\}}$ of random variables $\xi$ in the implementation of the hypothesis $H_{1}$ (there is a signal $S_{9}$ in the communication channel) and the alternative $H_{0}$ (there is no signal $S_{\vartheta}$ in the communication channel) will take the form of:

$$
\mathrm{F}\left(H_{0}\right)_{\mathrm{i}, \mathrm{j}\{k k\}}=m_{\mathrm{i}, \mathrm{j}\{k k\}}-m_{\mathrm{i}\{s k\}} m_{\mathrm{j}\{k k\}}, \mathrm{F}\left(H_{1}\right)_{\mathrm{i}, \mathrm{j}\{k k\}}=u_{\mathrm{i}, \mathrm{j}\{k k\}}-u_{\mathrm{i}\{k k\}} u_{\mathrm{j}\{k k\}},
$$

where $m_{\mathrm{i},\{s k\}} m_{\mathrm{i}\{k k\}}, u_{\mathrm{i}, \mathrm{j}\{k k\}}, u_{\mathrm{i}\{s k\}} i, j=\overline{1, s}$ are the ordinary moment of a random variable $\xi$ under the hypothesis and the alternative [6].

Polynomial DR has such form:

$$
\Lambda_{s(s k)}=\sum_{i=1}^{s} \mathrm{k}_{\mathrm{i}(\mathrm{s})\{s k\}} \sum_{v=1}^{n} x_{v}^{i}+\mathrm{k}_{0(\mathrm{~s})\{s k\}}
$$

whose optimal coefficients provide a fixed type I error probability $\alpha_{s n}$ with a minimized type II error probability $\beta_{s n}$ and are contained in the last value of the Neumann-Pearson instantaneous rate of the quality [6]:

$$
K u P(G, E)=\left[\frac{G_{0, s}\{k k\}}{(1-C)^{2}}+\frac{G_{1,\{s k\}}}{C^{2}}\right]\left[E_{1, s\{k k\}}-E_{0, s\{k k\}}\right]^{-2}
$$

where $E_{0, s\{k k\}}, E_{1, s\{k\}}$ and $G_{0, s\{s k\}}, G_{1, s\{s k\}}$ are the expectation function and variance of DR (1) within the hypothesis $H_{1}$ and the alternative $H_{0}$, which look like:

$$
\begin{gathered}
E_{0, s\{s k\}}=n \sum_{i=1}^{s} \mathrm{k}_{\mathrm{i}(\mathrm{s})\{\{k k\}} u_{\mathrm{i}(\mathrm{s})\{s k\}}, E_{1, s\{s k\}}=n \sum_{i=1}^{s} \mathrm{k}_{\mathrm{i}(\mathrm{s})\{s k\}} m_{\mathrm{i}(\mathrm{s})\{s k\}}, \\
G_{0, s\{k k\}}=n \sum_{i=1}^{s} \sum_{j=1}^{s} \mathrm{k}_{\mathrm{i}(\mathrm{s})\{s k\}} \mathrm{k}_{\mathrm{j}(\mathrm{s})\{s k\}} \mathrm{F}\left(H_{0}\right)_{\mathrm{i}, \mathrm{j}\{s k\}}
\end{gathered}
$$




$$
G_{0, s\{k k\}}=n \sum_{i=1}^{s} \sum_{j=1}^{s} \mathrm{k}_{\mathrm{i}(s)\{s k\}} \mathrm{k}_{\mathrm{j}(s)\{s k\}} \mathrm{F}\left(H_{0}\right)_{\mathrm{i}, j\{s k\}}
$$

The coefficients of DR (1), which are optimal by the instantaneous rate of the Neumann-Pearson description, are found by solving the simultaneous linear algebraic equations:

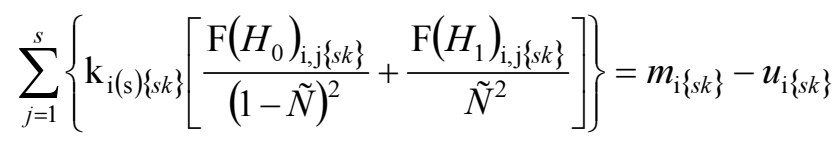

where $C$ is out of the fixed type I error probability of DR:

$$
\alpha_{2 s}=\frac{G_{0, s\{s k\}}}{(1-C)^{2}\left[E_{1, s\{s k\}}-E_{0, s\{s k\}}\right]^{2}}
$$

where the minimized fixed type II error probability of DR (1) has the following form:

$$
\beta_{2 s}=\frac{G_{1, s\{k k\}}}{C^{2}\left[E_{1, s\{s k\}}-E_{0, s\{s k\}}\right]^{2}}
$$

When the simultaneous estimation of the parameter of constant signal and the variance of the nonsymmetrical interference two equations are composed that maximize of the truncated stochastic polynomial of degree $S$ with the reduction depth complexity $\ell$ related to estimated parameters $\vartheta$ and $\chi_{2}$. For each of the equations it's necessary to calculate the coefficients $\mathrm{h}_{\mathrm{i}(\mathrm{s})\{s k\}\langle\ell\rangle[1]}, \mathrm{h}_{\mathrm{i}(\mathrm{s})\{\{s k\} \ell\rangle[2]}, i=\overline{1, s}$. The analytical expressions of these coefficients are found by solving the systems of simultaneous linear algebraic equations:

$$
\begin{gathered}
\sum_{j=1}^{s-\ell} \mathrm{h}_{\mathrm{i}(\mathrm{s})\{s k\}\langle\ell\rangle] 1]} K_{i, j\{s k\}}=\frac{\partial}{\partial \vartheta} m_{i\{k k\}}, i=\overline{1, s} \\
\sum_{j=1}^{s-\ell} \mathrm{h}_{\mathrm{i}(\mathrm{s})\{s k\}\langle\ell\rangle[1]} K_{i, j\{s k\}}=\frac{\partial}{\partial \chi_{2}} m_{i\{s k\}}, i=\overline{1, s} \quad i \neq(c, e, \ldots, l)
\end{gathered}
$$

where $K_{i, j\{s k\}}=m_{i+j\{s k\}}+m_{i\{s k\}} \cdot m_{j\{s k\}}$ is the centered correlate of nonsymmetrical random variables $\xi, m_{i\{k k\}}$ is the moment of the I order of the additive mixture of constant signal $S_{9}$ and the noise variance $\chi_{2}$. 
The coefficients $\mathrm{h}_{\mathrm{i}(\mathrm{s})\{s k\}\langle\ell\rangle[1]}, \mathrm{h}_{\mathrm{i}(\mathrm{s})\{\{k\}\} \ell \ell[2]} i=\overline{1, s}$ provide the minimum variance of the estimates $\hat{\vartheta}$ and $\hat{\chi}_{2}$, obtained by the method of simultaneous estimation, which is based on the methods of maximization of a polynomial [5] and on the maximization of the abridged stochastic polynomial.

On the fourth and fifth stages, the detection of joint signal $S_{9}$ and estimation of its parameter $\vartheta$ are conducted. The ratio "signal/noise" $q=S_{\vartheta}^{2} / \chi_{2}$ and the type I error probability $\alpha$ is set for nonsymmetrical additive noise $\eta$.

In the process of detection of a constant signal $S_{9}$ the algorithm "sliding window" is applied, and the width of the window is determined. The optimal width of the sliding window is the case when the number of samples of the window $n$ coincides with the number of samples, which the analyzed signal $S_{9}$ takes with duration $T$.

When the condition for DR $\Lambda_{s(s k)}>0$ is realized, the hypothesis $H_{1}$ becomes credible, which indicates the presence of the tested signal $S_{9}$ in the communication channel; otherwise, the decision about the realization of the hypothesis $H_{0}$ is made, which corresponds to the presence of the only interference (noise) $\eta$ in the communication channel.

Based on the proposed method in [6] the synthesis of linear $(s=1)$ and nonlinear DR $(s \geq 2)$ is realized. In Figure 2 the dependence of value changes of the synthesized DR $\Lambda_{3(s k)}$ with $s=3, q=0,8, n=100, \alpha_{3 s}=10^{-3}$ (hereinafter all the experimental results are obtained at the same parameters).

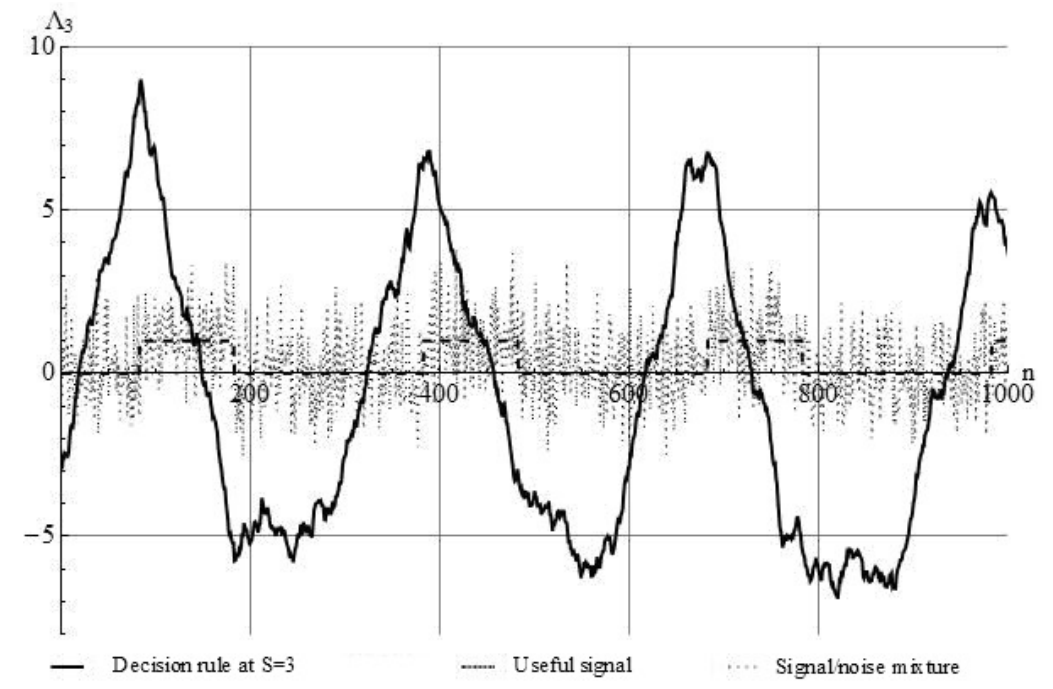

Fig. 2. The value change of the polynomial DR 
As it is shown in Figure 2, the maximum value of DR coincides with the time moment when the beginning of the sliding window coincides with the signal $S_{9}$. When making the hypothesis about the presence of a signal $S_{9}$ in the communication channel, the counts of the sampled values $x_{n}$ are determined and the time of arrival $\tau$ is calculated.

The next step of computer simulation modeling is the implementation of the evaluation process of the signal $\vartheta$ parameter. First, the equation of maximizing the polynomial for the signal parameter and the equation of maximizing the abridged polynomial with the parameter of the depth truncation $\ell$ for the variance of nonsymmetrical interference.

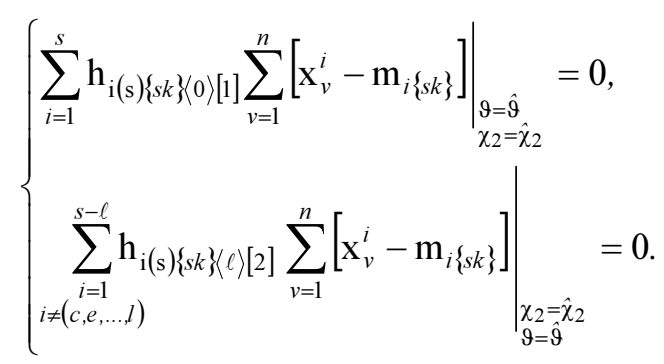

Then we solve the simultaneous equations (3) and select one pair of radicals on the criteria of the maximum amount of information about the parameter $\vartheta$. Further the analysis of the obtained results is applied. The variance of simultaneous estimation of the parameter of signal $\vartheta$ and the variance of noise $\chi_{2}$ are compared with the results obtained by classical methods. The decrease in the variance of the simultaneous estimation is described by the coefficient $g_{3,1}\left(\gamma_{3}\right)$ [5].

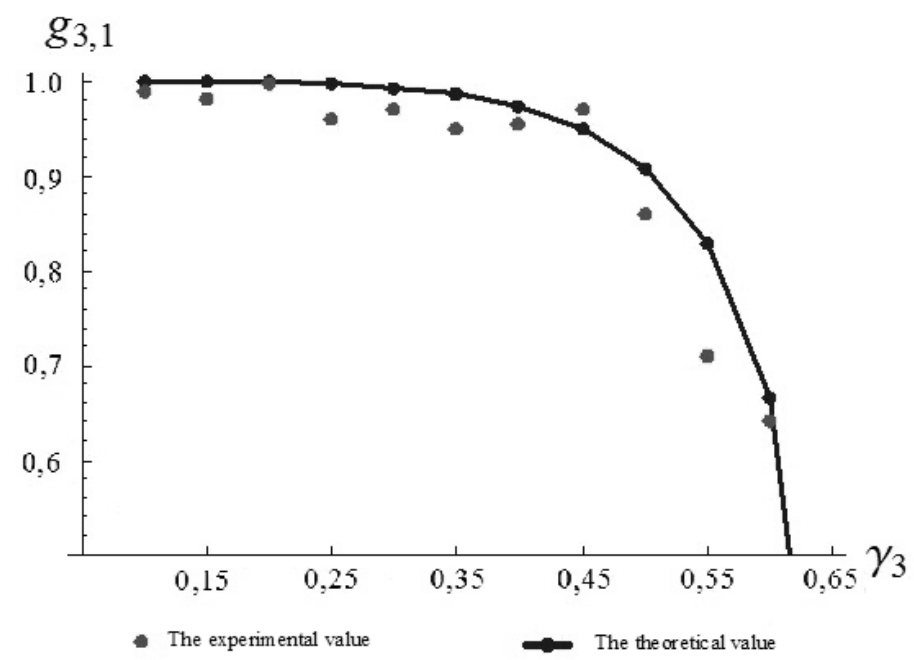

Fig. 3. The dependence of the reduction coefficient of the variance 


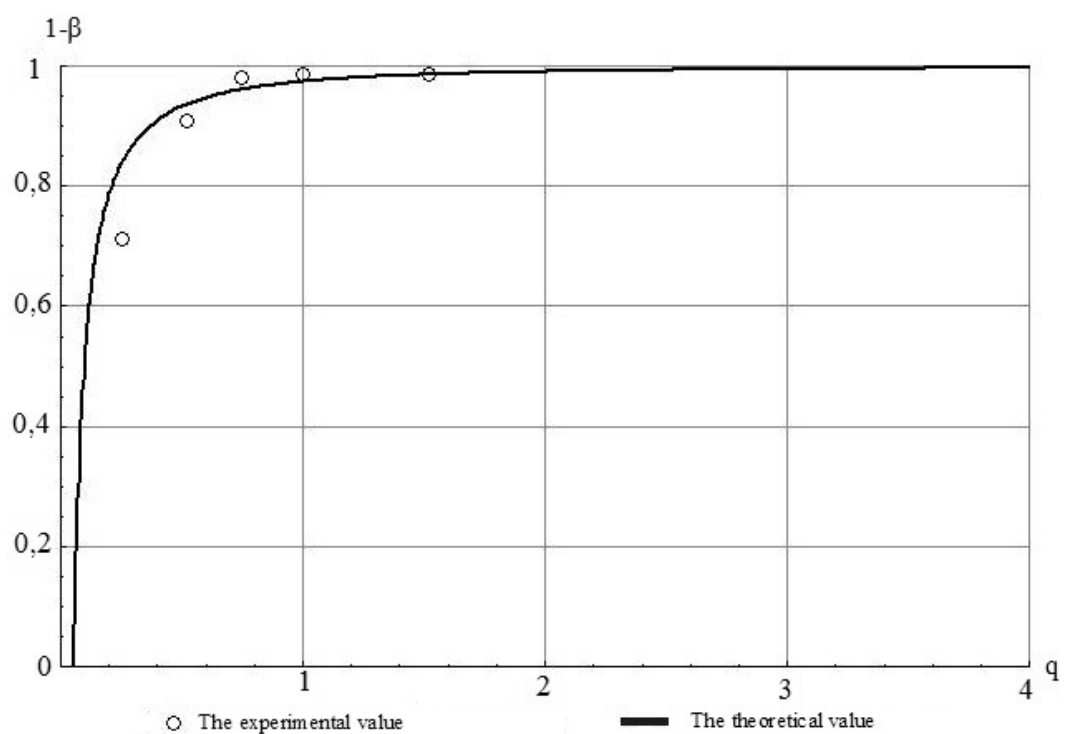

Fig. 4. The dependence of the probability of correct detection of the signal

The theoretical and experimental values of the coefficient of the variance reduction $g_{3,1}\left(\gamma_{3}\right)$ and probability of correct detection $1-\beta$ are compared in Figures 3 and 4.

\section{Conclusions}

The results of the simulation modeling experimentally confirmed the theoretically obtained results, which show that the use of the moment-cumulant description of non-Gaussian random values, polynomial DR, abridged stochastic polynomials for estimating the parameters of signals and noise allows one to increase the accurate characteristics in the form of reduction of the estimate variance and increase of the probability of correct detection of the assessment systems and the detection signals compared with known results.

\section{References}

[1] Van Trees H.L., Detection, Estimation, and Modulation Theory, Van Trees H.L., Part IV, Optimum Array Processing, John Wiley, 2002, 1470 p.

[2] Bezruk V., The theoretical bases of designing the systems of recognition of signals for the automated radio monitoring: the monograph, Bezruk V., Pevtsov G.X., Collegium, 2007, 430 p.

[3] Shelukhin O., The non-Gaussian processes, O. Shelukhin, I. Belyakov, Politechnica, St. Petersburg 1992, $312 \mathrm{p}$.

[4] Malakhov A., The Cumulant Analysis of the Non-Gaussian Processes and Their Transformations, The Soviet Radio, M. 1979, 376 p. 
[5] Kunchenko Y.P., Polynomial Parameter Estimations of Close to Gaussian Random Variables, Shaker Verlag, Aachen 2002, 396 p.

[6] Lega Y., The construction of the polynomial decision rules on the Neumann-Pearson instantaneous rate for the test of the statistic hypothesis, Electronics and Control Systems 2008, 4(18), $5-12$. 